The final version of this paper has been published in European Journal of Cardiovascular Nursing, online 13 February 2013, by SAGE Publications Ltd. All rights reserved. (C) Anne McBride, Lorraine Burey, Margo Megahed, Carolyne Feldman, and Christi Deaton. The online version of this article can be found at:

http://cnu.sagepub.com/content/early/2013/04/22/1474515113478531

\title{
The role of patient-held alert cards in promoting continuity of care for Heart Failure Patients
}

Anne McBride ${ }^{\mathrm{a}^{*}}$, Lorraine Burey ${ }^{\mathrm{b}}$, Margo Megahed ${ }^{\mathrm{c}}$, Carolyne Feldman $^{\mathrm{c}}$, Christi Deaton ${ }^{\mathrm{d}}$

${ }^{a}$ Manchester Business School, The University of Manchester, Booth Street West, Manchester, M15 6BP, UK

${ }^{\mathrm{b}}$ National Institute of Health Research (NIHR) Collaboration for Leadership in Applied Health Research and Care (CLAHRC) for Great Manchester, Salford Royal NHS Foundation Trust, Stott Lane, Salford M6 8HD, UK

${ }^{\mathrm{c}}$ University Hospital of South Manchester NHS Foundation Trust, Southmoor Road, Wythenshawe, Manchester, M23 9LT, UK

${ }^{\mathrm{d}}$ School of Nursing, Midwifery \& Social Work, The University of Manchester, Jean McFarlane Building, Oxford Road, Manchester, M13 9PL and Central Manchester University Hospitals NHS Foundation Trust, Manchester Royal Infirmary, Oxford Road, Manchester, M13 9WL, UK

* Corresponding author. Manchester Business School, The University of Manchester, Booth Street West, Manchester, M15 6BP, UK. Tel.: +44

(0) 161306 5863; fax: +44 (0) 1613063505.

E-mail address: a.mcbride@ manchester.ac.uk 


\begin{abstract}
Background: Patients with heart failure (HF) managed by community HF specialist nurses (CHFSNs) may have episodes of (often unrelated) ill-health managed separately in hospital. Inadequate communication and multi-disciplinary working between these different providers can impact on the effectiveness of care.

Aim: This service improvement project explored the potential of patient-held alert cards to improve communication and continuity of care for $\mathrm{HF}$ patients moving between CHFSNs and hospital settings.

Methods: Alert cards were distributed to 119 patients on a community case load for presentation at hospital or emergency department. Follow up data were obtained from practitioners and patients at 12 months.

Results: At 12 months, 38 patients from the CHFSN caseload experienced 61 hospital admissions. CHFSNs were informed of $80 \%$ of admissions by practitioners $(61 \%)$ and family members (38\%). They were also informed of $59 \%$ of discharges. Notification of admission by hospital staff increased from zero in the previous 12 months, to 19 notifications. CHFSNs were more involved with hospital care, and patients reported increased confidence with the alert cards.

Conclusions: The study has shown that alert cards can increase the involvement of CHFSNs in the ongoing care and discharge planning process. They can also empower patients and carers to take an active role in their own care.
\end{abstract}

Key words:

Heart failure, co-morbidity; continuity of care; patient-held alert card, multidisciplinary working

\title{
Introduction
}

Heart failure (HF) has significant implications for both patients and health systems. In the UK, it affects around 750,000 people, with an estimated 27,000 new cases each year ${ }^{1}$. It accounts for $2 \%$ of in-patient days in the National Health Service (NHS) ${ }^{2}$ and between 4 and $5 \%$ of all admissions to hospital ${ }^{3}$. In addition, co-morbidities such as respiratory conditions, renal dysfunction, anaemia, arthritis, cognitive dysfunction, diabetes and depression ${ }^{4}$, increase the possibility of hospital admissions. This has implications for older HF patients who may have in excess of five co-morbidities 5 . Not only do co-morbidities contribute to the development and progression of HF, they also complicate patient management ${ }^{6,5}$. Whilst HF-specific research indicates the use of optimal medical therapy ${ }^{6}$, heart failure specialist nurses ${ }^{7,8}$, and multi-disciplinary discharge and follow-up ${ }^{2}$ to improve patient outcomes, with some exceptions ${ }^{6}$, much research and guidance for practice tends to be single-disease specific and assumes an initial or primary diagnosis of HF. It does not allow for practitioners prioritising other 
co-morbidities. In addition, admissions for co-morbidities could be managed by professionals who may not be HF-oriented ${ }^{9}$, may not have knowledge of the patients' medical management from other providers, or may be insufficiently knowledgeable of the polypharmacy associated with multiple chronic conditions ${ }^{5}$. In addition, they may not be aware of the extent to which older HF patients benefit from comprehensive discharge planning and follow-up ${ }^{10,11}$. There is a risk, therefore, of communication and co-ordination failure in the hospital admission of $\mathrm{HF}$ patients. This is symptomatic of a wider issue of ensuring continuity of care for patients with concomitant chronic conditions in transition between healthcare providers and/or between generalists and specialists ${ }^{12,13,14}$ and where the literature indicates that patients and families often assume that providers know what has happened before ${ }^{14}$.

Improving the transition of HF patients through the community-hospital interface was one stream of work conducted within the National Institute for Health Research (NIHR) Collaboration for Leadership in Applied Health Research (CLAHRC) for Greater Manchester (GM). Greater Manchester has one of the lowest life expectancies in England and cardiovascular disease is the single most important source of morbidity and premature mortality in the conurbation. Bringing together academic institutions, NHS healthcare providers and commissioners, the mission of GM CLAHRC is to improve healthcare and reduce health inequalities for people with cardiovascular conditions ${ }^{15}$. Implementation within GM CLAHRC is based on four building blocks ${ }^{15}$. First, recognising that successful implementation of research into practice is dependent on the complex interplay of evidence, context of implementation and facilitation ${ }^{16}$. Second, that implementation can be operationalised through small, incremental changes ${ }^{17}$. Third, a multi-professional team approach which includes a clinical lead, academics with healthcare management expertise and knowledge transfer associates (KTAs) who act as main facilitators of change in the field. Fourth, the embedding of evaluation and learning within any research into practice initiative.

\section{Methods}

The following sections indicate how the principles of GM CLAHRC were applied to improve the management of HF patients admitted to hospital from a community HFSN (CHFSN) caseload. The first section identifies how the context was mapped to provide an understanding of the patient journey between hospital and community services, and reports subsequent work with stakeholders to determine possible solutions. The second section indicates the changes made to practice during the pilot and the nature of the evaluation. 


\section{Context mapping and identifying potential for change}

The context in which HF patients were managed was systematically mapped by the KTAs through two stakeholder meetings, 27 interviews with practitioners and commissioners, an audit of 50 patient records, and analysis of patient data. Despite examples of innovative and evidence-based practice, areas for improvement existed at the community-hospital interface. Interviews revealed that community nurses were not notified when HF caseload patients were admitted or discharged. Ward staff reported uncertainty in relation to contacting community health care practitioners (HCPs) about their patients. Patient care was impacted by unnecessary tests during hospitalisation, changes to HF medications, and limited specialist input into discharge planning.

The mapping exercise was presented to a stakeholder meeting and led to a mandate to develop a system that identified community-managed HF patients on admission to secondary care. Two CHFSNs agreed to test the changes on their caseload. Context mapping indicated that flagging patient records - physically or electronically - would either not be adequate or possible. Patient-held alert cards were considered as they could provide important information to HCPs, patients and carers. Although there is limited evaluation of the usefulness of such cards in enhancing continuity of care ${ }^{13}$, patients may feel empowered by the possession of such cards ${ }^{18,19}$. A search of grey literature - and subsequent contact - indicated that modification of an alert card used by a quality improvement team at North Cumbria University Hospitals NHS Trust could enable the desired changes to be made.

\section{Making changes and embedding evaluation}

In accordance with the principles of the Model of Improvement ${ }^{17}$ (adopted within GM CLAHRC $^{15}$ ), improvements were to be achieved through a small set of incremental changes which were facilitated by the KTAs. The first three changes were made by the team, with the expectation that they would lead to the latter two changes by others. In the first instance, the team developed a card. This involved discussions with HCPs and patients. A prototype card received positive feedback from HCPs, and patient feedback was incorporated into the design of the card. The card (see Figure 1) is laminated, the size of a credit card and designed to fit into a wallet or purse. The card indicates the carrier has HF. It references the last echocardiogram (ECHO) and provides data on ejection fraction and left ventricular (LV) function. These were included because it is helpful to know in non-HF admissions whether a patient has left ventricular systolic dysfunction (LVSD) or heart failure with preserved ejection fraction (HFPEF) and their EF, as this can influence management during the inpatient stay and alert the provider to potential risks and prognosis. The card also includes 
contact details of GP and CHFSN and an instruction to contact the CHFSNs in case of hospital admission.

Figure 1 Heart Failure Alert Card
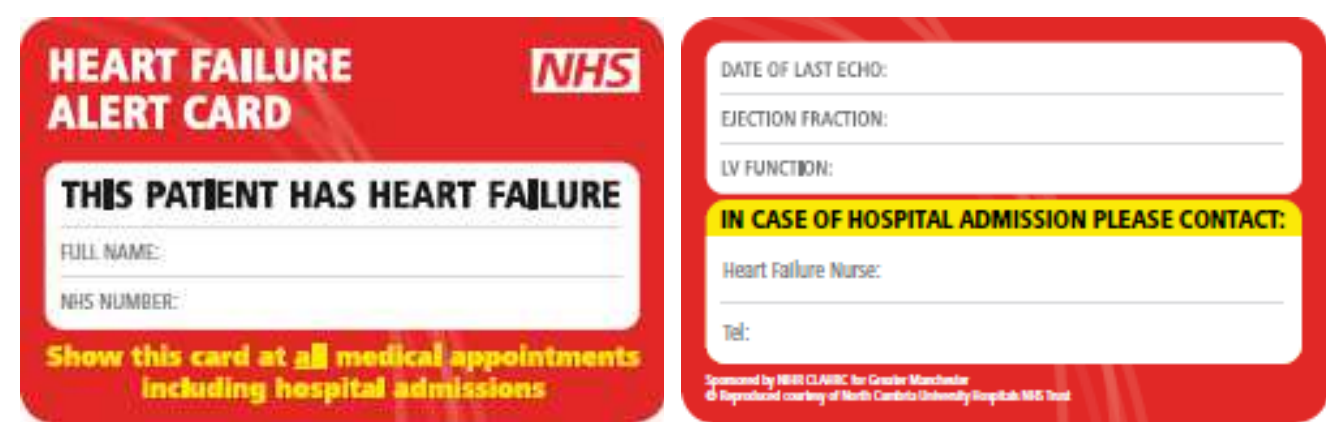

Second, the card required circulation and explanation to patients. The card was distributed $\mathrm{t}$ o patients on a CHFSN caseload for a period of 12 months from September 2010 to September 2011. Most cards were issued by the CHFSNs in faceto-face appointments with an explanatory leaflet and verbal reinforcement. Third, explanatory leaflets were circulated to the HF link nurse and ward staff at the local hospital with verbal reinforcement at ward level. The fourth anticipated change was that patients would present the card to HCPs and, if admitted to hospital, would ask staff to inform the CHFSNs. The fifth change required hospital staff to contact the CHFSNs and involve them in subsequent patient management. As indicated in Table 1, it was anticipated that these latter two changes would lead to improvements within the six domains of quality identified by the Institute of Medicine $^{20}$ : patientcenteredness, safety, effectiveness, efficiency, timeliness and equity, and methods were established to evaluate this potential. As this was an audit and service improvement programme, no ethical permission was sought for the project. Evaluation of the project was conducted by the KTA. Patients were asked if they were willing to participate in a telephone interview and provided verbal consent. Three HCPs, two patients and one carer were interviewed as part of the evaluation. 
Table 1 Identification of proposed changes, desired improvements and methods of evaluation

\begin{tabular}{|c|c|c|}
\hline Proposed changes & $\begin{array}{l}\text { Desired improvements in } \\
\text { dimensions of quality }\end{array}$ & Methods of evaluation \\
\hline $\begin{array}{l}\text { Patient/family retains } \mathrm{HF} \\
\text { alert card and presents on } \\
\text { hospital admission }\end{array}$ & $\begin{array}{l}\text { Patient-Centeredness } \\
\text { Care is more appropriate to patient } \\
\text { needs. } \\
\text { Empowerment of patient/carers }\end{array}$ & $\begin{array}{l}\text { Qualitative Data } \\
\text { patient and carer interviews } \\
\text { (usage and experience of card) } \\
\text { HCP interviews } \\
\text { (experience, examples of impact, } \\
\text { clinical outcomes) }\end{array}$ \\
\hline $\begin{array}{l}\text { On presentation of } \mathrm{HF} \\
\text { alert card, hospital } \\
\text { contacts and involves } \\
\text { CHFSN on admission } \\
\text { /discharge. }\end{array}$ & 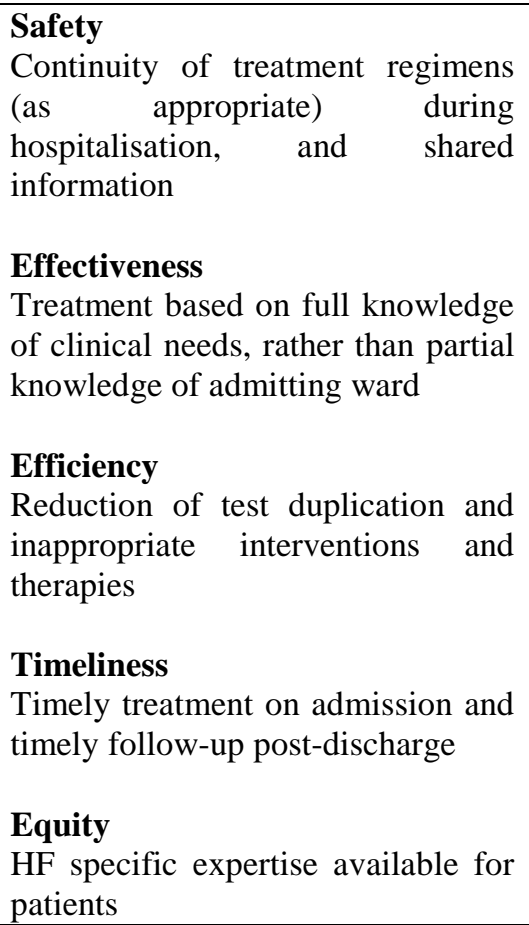 & $\begin{array}{l}\text { Quantitative Data } \\
\text { Baseline data (previous } 12 \\
\text { months); data collected at } 6 \text { and } 12 \\
\text { months: } \\
\text { CHFSN caseload } \\
\text { Admissions } \\
\text { Reason for admission } \\
\text { Notification of patient admission } \\
\text { Notification of patient discharge } \\
\text { Status of notifier }\end{array}$ \\
\hline
\end{tabular}

\section{Results}

During the pilot, 160 patients, all of whom were over the age of 40 with $50 \%$ over 80 , were managed by the CHFSNs. Fifty-one percent were female, with patients having an average of three co-morbidities with chronic kidney disease $(25 \%)$ the most prevalent. Most of the patients (54\%) were NYHA class III, 26\% were class II, and 
4\% were class IV. Alert cards were issued to 119 patients. The CHFSNs excluded those patients who were cognitively impaired or frail. Table 2 provides an overview of caseload admission activity during the 12 month pilot study. Three key changes are presented below with quantitative data and quotations from interviews.

Table 2 CHFSN caseload and admission activity during 12 month pilot project

\begin{tabular}{|l|l|}
\hline Activity & Volume \\
\hline Patients treated & 160 \\
\hline Patients issued with alert cards & $119(75 \%$ of patients treated $)$ \\
\hline All hospital admissions from caseload & 61 (38 patients) \\
\hline Unscheduled admissions & $59(97 \%$ of admissions $)$ \\
\hline Single admissions & $48(80 \%$ of admissions $)$ \\
\hline Admissions due to HF from the caseload & $7(11 \%$ of admissions $)$ \\
\hline Notification of admission to CHFSN & $\begin{array}{l}49(80 \% \text { of admissions }) \\
30 \text { by HCPs (19 hospital staff; } 11 \\
\text { community nurses }) \\
19 \text { family notification }\end{array}$ \\
\hline
\end{tabular}

\section{Increased community-hospital contact on admission/discharge}

The contact between hospitals and CHFSNs regarding caseload admissions increased significantly during the HF alert card pilot study. In the previous 12 months, hospital staff had not notified CHFSNs of any caseload admissions. This compares with hospital staff contact in 19 of the 49 notifications of admission. This increase in contact is particularly important because HF was the reason for only $11 \%$ of admissions and 59 of the 61 admissions (97\%) were not to a specialist cardiology ward. Indeed, it was this cohort of patients admitted to general medical wards whom the CHFSNs had previously identified as being at a higher risk of having their medication changed or stopped.

Patient interviews echoed the concern that hospital staff needed to know as much as possible about them:

"Personally I thought it was a good idea, that your information is available to all. When I went into hospital, it took quite a long time to diagnose what was wrong with me ... so on that basis I think it's a good idea". 
Overall, 30 notifications of admission came from HCPs $(61 \%)$. This also improved communications within the hospital as patient admissions were notified to a specialist HF link nurse:

"It lets them [ward staff] know that they're heart failure and they can perhaps contact me.".

Another benefit indicated by the CHFSNs is that they were notified of hospital admissions outside the catchment area:

"I've had one or two patients admitted into hospital as a result of a fall but in different parts of the country and they've produced the card and we were contacted by the hospital".

Presentation of the card in outpatient clinics has also led to CHFSNs receiving copies of letters previously only sent to the GP.

The CHFSNs were informed when their caseload patient was due for discharge in 36 of the 61 admissions. Whilst not infallible, this compares favourably with zero notification in the previous 12 months. Increasing notification of admission during the pilot study enabled CHFSNs to start communicating with the HCPs who would be involved in the discharge:

"... I used to just ring the wards and ask them to tell me when the patient was going home ... I now automatically speak to the SHO [Senior House Officer] as the person looking after them. Also, that's the chance to determine some of the decision-making."

\section{Involvement of CHFSNs facilitates continuity of care for HF patients}

It was expected that increased hospital contact would facilitate continuity of care for $\mathrm{HF}$ patients. Interviews with the CHFSNs revealed a number of ways in which increased contact had improved different dimensions of care. First, the contribution of continuity of care to safety, effectiveness, timeliness and equity of treatment is indicated through these experiences:

"...we were able to highlight to the staff that a particular patient needed quite a high dose of diuretic or to make sure that they were restarted on a beta-blocker if it had been stopped" and "I certainly have had phone calls from registrars in the hospital to say this patient is under your care and there were some issues around 
medication and I've discussed it, particularly the high risk patients, on very, very, high doses of medication that we don't want to dramatically reduce."

In addition, CHFSNs cited earlier examples where medications had been changed by hospital doctors, with changes not being consistent with HF guidelines or the patient's HF management needs. This resonates with extant literature which indicates the incidence of patients receiving contradictory advice in hospital ${ }^{5}$ and reinforces the importance of the increased involvement of CHFSNs whilst patients from their caseload are in hospital.

Second, improved efficiency can be seen in the example of one patient who was repeatedly referred to Accident and Emergency (A\&E) by the out-of-hours service due to high potassium blood levels. Following two previous admissions, on subsequent occasions she showed her HF alert card and avoided being admitted as an emergency. A\&E staff were assured that the patient would be followed up by the CHFSN who was aware of her condition. Efficiency was also indicated through the sharing of information, such as a community ECHO, to save repeating one in the hospital.

Third, the HF alert card also enabled continuity of care within primary care:

"A lot of GPs don't have access to echocardiogram results so although patients have an ECHO they don't have any results, they don't know when the ECHO was done, so on the card itself it states the date of the last ECHO and it also states the ejection fraction."

\section{Alert cards empowered patient/carers to take more active role in care}

The alert cards empowered both patients and carers to take a more active role in their care. Firstly, although CHFSNs explain a HF diagnosis as part of their assessment, the card itself appeared to prompt patients to ask questions about their condition,

"I have had a few that have wanted to know what things meant on the card, particularly when it says severe LVSD [left ventricular systolic dysfunction].

Second, family members notified CHFSNs of hospital admission in 19 cases (38\% of admissions), as

"the alert card is a reminder to them that they are not to forget that even if it's not anything to do with their heart, they're to get in touch with us." 
Third, HCPs indicated how the cards seemed to give families and carers 'permission' to call them if they were unsure of anything:

"we do encourage families, but maybe it gave them a bit of ownership and a bit of being able to say there is a problem here ... it kind of makes it easier in some ways."

Lastly, the cards gave patients confidence in their interactions with hospital staff, with one patient noting,

"It was just that I feel more confident with it now because it's better than me saying to them, 'look I've got heart failure'. It feels better handing them a card. It's more official looking."

On one occasion, this interviewee had challenged a nurse who had said she wouldn't be able to contact her CHFSN because there was no number on the card,

"I said yeah, there is a number on it, 'cause I just felt she didn't read it carefully".

Another patient indicated,

"If my tablets were changed, I would be showing them the card ..."

\section{Discussion}

This service improvement project underlines the issues of unscheduled hospital admissions, repeat admissions and co-morbidity of HF patients and reinforces the importance of continuity of care across the community-hospital interface ${ }^{12,13}$. Three types of continuity of care have been identified in every discipline - informational, management and relational ${ }^{14}$. In this study, relational continuity is achievable through the management of HF patients by a core team of CHFSNs. Hospital admission of community managed HF patients, however, threatens management continuity as it introduces the potential of providers to work in contradiction to each other ${ }^{14}$. Context mapping indicated the presence of management discontinuity, which was often linked to a break in informational continuity as information did not necessarily follow the patient. Thus, whilst evidence may exist to assist HCPs in their management of heart failure $^{6,21}$ it requires non-specialist HCPs to be aware that a presenting patient has HF, and that this condition may already be actively managed by a community-based nurse. 
Without this informational and management continuity, care is in danger of not being patient-centred, safe, effective, efficient, timely or equitable regardless of need.

In the absence of immediate technological solutions to bridge the primary and secondary interface ${ }^{12}$, this study illustrated how a patient-held alert card can provide informational continuity as it encouraged general wards to liaise with specialist nurses on admission/discharge to ensure the continuation and appropriateness of care. In essence, this card stimulated communication across the interface ${ }^{12}$ and contributed to management continuity. It is difficult to improve outcomes in the multimorbid population but interventions which focus on particular risk may be more effective $\mathrm{e}^{22}$. A particular risk for HF patients admitted into hospital is that key drug regimens may not be maintained or that titration periods may be interupted. Whilst not eliminated, this risk is lowered by providing a means through which specialist nurses and hospital HCPs can provide shared care of the patient. Although a systematic review of shared care interventions in chronic disease management provides mixed results, it does indicate a clear improvement in prescribing in the studies that considered this outcome $^{23}$. Enabling patient-centred prescribing during hospital admission is of particular pertinence when successive HF audits link suboptimal prescribing with higher mortality rates ${ }^{3}$.

This project confirms earlier work that indicates that patient-held medical cards can contribute to continuity of care ${ }^{13,14}$ beyond the immediate, emergency needs traditionally associated with alert cards ${ }^{24}$. An essential element in the effectiveness of this intervention was the action of patients and carers in both retaining and presenting the card to HCPs. The patient, carer and HCP interviews reinforced earlier findings ${ }^{18,19}$ that patients welcome such cards and feel empowered by them. The 'official' nature of the card appeared to give them confidence in their interactions with secondary care HCPs, along with the instructions written on the card (verbally reinforced by the CHFSNs). The cards also prompted patients to ask CHFSNs about the information on the card which, in turn, could lead to a beneficial individualisation of patient education ${ }^{25}$. Such patient-prompted questions are important as it is possible that HF patients do not appreciate the seriousness of their condition ${ }^{26}$ or may find the information given to them at the time of diagnosis difficult to understand ${ }^{27}$. The increased involvement of carers in alerting CHFSNs of hospital admissions during the pilot study is also important as evidence suggests that caregivers of people with HF often experience caregiver burden and emotional distress ${ }^{28}$, with this burden lowering when a partner of a HF patient experiences higher control over the heart disease ${ }^{29}$.

This study reinforced the importance of understanding the context in which HCPs work and reinforced earlier work that successful implementation of research into practice is dependent on the complex interplay of evidence, context and facilitation ${ }^{15}$. Considerable efforts have been put into conducting research which indicates the beneficial use of particular HF treatment regimens, ${ }^{6,21} \mathrm{HFSNs}^{7,8}$ and multi- 
disciplinary discharge and follow-up ${ }^{2}$. This study provides an illustration, however, that good research is a necessary but not sufficient condition to guarantee its uptake ${ }^{15}$. Informational continuity, for example, is a necessity too. By mapping the organizational context in which implementation is to take place, and understanding key stakeholders and their roles and relationships, the GM CLAHRC KTAs were able to develop a series of small, incremental changes which had the potential to be both acceptable to participants and meet the desired improvements outlined in Table 1. Thus, whilst modest in technological terms, the development of this hand-held, nonelectronic card fitted the context in which it was being introduced and led to actions by patients, carers and HCPs which improved informational continuity and impacted on management continuity.

Lastly, whilst management continuity is of particular importance to HF patients it is also a concern for generalists and specialists managing any chronic disease management or patient with multimorbidity. Developing an HF alert card still only focuses on a single-disease. GM CLAHRC is now reflecting on the potential for a 'multi-card' to address cardiovascular multimorbdity.

\section{Conclusion}

This pilot project demonstrated that a simple device, a patient held alert card can improve communication and coordination between CHFSNs and hospital staff, and empower patients and families. Health care services remain largely fragmented, however, and pose many obstacles to providing integrated care for patients with multiple long-term conditions. Other innovative solutions need to be developed and tested to improve continuity of care for patients. For those solutions which are found to be effective, implementation strategies need to be robust and evidence-based to enable change in practice.

\section{Funding}

This work was funded by the CLAHRC for Greater Manchester, which is jointly funded by National Institute of Health Research (NIHR) and primary care trusts in Greater Manchester.

\section{Acknowledgements}

We would like to thank the reviewers for their helpful suggestions. We would also like to thank University Hospital of South Manchester NHS Foundation Trust and Simone Reece, Advancing Quality heart failure nurse for supporting this project. 
Thanks also to Stephanie Tierney and Rachel Lewis for conducting interviews, and Ruth Boaden, Deputy Director GM CLAHRC, Joanne Thomas, GM CLAHRC Implementation Programme Manager and Catherine Deering, Information Specialist. The views and opinions in this paper do not necessarily reflect those of the NHS, the NIHR or the Department of Health.

\section{Conflict of interest}

The authors have no conflict of interest to report.

\section{References}

1. British Heart Foundation. Our heart disease facts, http://www.bhf.org.uk/media/news-from-the-bhf/bhf-facts.aspx; 2012; Available from: http://www.bhf.org.uk/media/news-from-the-bhf/bhf-facts.aspx, accessed 23 August 2012.

2. Bernard S, Brodie E and Lohr K. Healthcare delivery models for heart failure. Report for The Health Foundation, 2007. London: The Health Foundation.

3. NICOR: National Institute for Cardiovascular Outcomes Research. National Heart Failure Audit, April 2010 - March 2011, Report for Healthcare Quality Improvement Partnership. Available at: http://www.hqip.org.uk/national-heartfailure-audit/ (2012), accessed 17 July 2012.

4. Sutherland K. Bridging the quality gap: heart failure. Report for The Health Foundation, 2010. London: The Health Foundation.

5. De Geest S, Steeman, E, Leventhal ME et al. Complexity in caring for an ageing heart failure population: concomitant chronic conditions and age related impairments. Eur J Cardiovasc Nurs 2004; 3: 263-270.

6. McMurray JJV, Adamopoulos S, Anker SD et al. ESC Guidelines for the diagnosis and treatment of acute and chronic heart failure 2012. Eur Heart J 2012; 33: 1787-1847.

7. Blue L, Lang E, McMurray JJV et al. Randomised controlled trial of specialist nurse intervention in heart failure. Br Med J 2001; 323: 715-8.

8. Pattenden J, Coulton S, Spilsbury K et al. The Development and Impact of the British Heart Foundation and Big Lottery Fund Heart Failure Specialist Nurse Services in England. Report for British Heart Foundation, 2008. London: British Heart Foundation.

9. Rushton C, Satchithananda DK and Kadam UT. Comorbidity in modern nursing: a closer look at heart failure. Br J Nurs 2011; 20(5): 280-XX 
10. Phillips CO, Wright SM, Kern DE et al. Comprehensive discharge planning with postdischarge support for older patients with congestive heart failure: A metalanalysis. JAMA 2004; 291: 1358-1367.

11. Yu DSF, Thompson DR, Lee DTF. Disease management programmes for older people with heart failure: crucial characteristics which improve post-discharge outcomes. Eur Heart J 2006; 27: 596-6122.

12. Kvamme OJ, Olesen F, and Samuelsson M. Improving the interface between primary and secondary care: a statement from the European Working Party on Quality in Family Practice (EQuiP). Quality in Healthcare 2001; 10: 33-39

13. Olola CH, Rowan B, Narus S, et al. Implementation of an Emergency Medical Card and a Continuity of Care. Methods Inf Med 2009; 48: 519-530.

14. Haggerty JL, Reid RJ, Freeman GF et al. Continuity of care: a multidisciplinary review. Br Med J 2003; 327 (7425): 1219-1221.

15. Harvey G, Fitzgerald L, Fielden S et al. The NIHR collaboration for leadership in applied health research and care (CLAHRC) for Greater Manchester: combining empirical, theoretical and experiential evidence to design and evaluate a largescale implementation strategy. Implement Sci 2011; 6:96

16. Kitson A, Rycroft-Malone J, Harvey $G$ et al. Evaluating the successful implementation of evidence into practice using the PARIHS framework: theoretical and practical challenges. Implement Sc 2008. 3:1.

17. Langley GJ, Nolan KM, Norman CL et al. The Improvement Guide 1996. San Francisco: Jossey-Bass.

18. Flavin S, Evans J \& Shapcott J. Proposal for a patient safety alert card to risk of pressure damage. J Tissue Viability 2009; 18(4):119.

19. Islam S, Robertson B, Fulkes $\mathrm{V}$ et al. A pilot survey of patient's perception of a special transfusion card - An inner city teaching Hospital experience. Conference: 29th Annual Scientific Meeting of the British Blood Transfusion Society Glasgow United Kingdom Transfus Med 2011; 21, Suppl. 1: 41.

20. Institute of Medicine. Crossing the Quality Chasm: A New Health System for the $21^{\text {st }}$ Century. Washington, DC: National Academies Press, 2001.

21. NICE: National Institute for Clinical Excellence. Chronic heart failure: management of chronic heart failure in adults in primary and secondary care (CG5), 2003. London: NICE. Available at www.nice.org.uk

22. Smith SM, Soubhi H, Fortin M et al. Interventions for improving outcomes in patients with multimorbidity in primary care and community settings. Cochrane Database Syst Rev 2012, April 18; 5:CD006560.

23. Smith SM, Allwright S, O'Dowd T. Effectiveness of shared care across the interface between primary and specialty care in chronic disease management. Cochrane Database Syst Rev 2007; July 18; (3): CD004910. 
24. Gooptu B, Ward L, Ansari SO, et al. Oxygen alert cards and controlled oxygen: preventing emergency admissions at risk of hypercapnic acidosis receiving high inspired oxygen concentrations in ambulances and A\&E departments. Emerg Med J 2006; 23: 636-638.

25. Fredericks S, Beanlands $\mathrm{H}$ et al. Effects of the characteristics of teaching on the outcomes of heart failure patient education interventions: a systematic review. Eur J Cardiovasc Nurs 2010; 9(1): 30-7.

26. Allen LA, Yager JE, Funk MJ et al. Discordance between patient-predicted and model-predicted life expectancy among ambulatory patients with heart failure. JAMA 2008; 299: 2533-42.

27. Banerjee P, Gill L, Muir V et al. Do heart failure patients understand their diagnosis or want to know their prognosis? Heart failure from a patient's perspective. Clin Med 2010; 10(4): 339-343.

28. Pattenden JF, Roberts $\mathrm{H}$ and Lewin RJ. Living with heart failure; patient and carer perspectives. Eur J Cardiovasc Nurs 2007; 6(4): 273-9.

29. Agren S, Evangelista L and Stromberg A. Do partners of patients with chronic heart failure experience caregiver burden? Eur J Cardiovasc Nurs 2010; 9(4): 254-62. 